Abstract: We present the results of a theoretical study providing details of propagation of laser radiation within disperse randomly inhomogeneous intermediately single-to-multiple scattering media. A quantitative analysis of scattering orders in the transition from single to multiple scattering is presented. Crossed source-detector fiber optics geometry used to separate the intensity of single scattering from higher scattering orders. The results demonstrate good agreement between analytical and Monte Carlo techniques. This validates the use of the Monte Carlo approach in the intermediate single-to-multiple scattering regime. The method used can be applied to verify analytical results against experiment via the Monte Carlo calculations that include imperfections of the experiment.

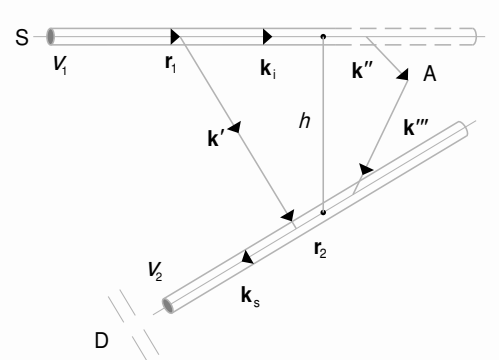

Schematic illustrating the experimental/computational geometry used to account for single-to-higher orders laser scattering

\title{
Low and high order light scattering in particulate media
}

\author{
I.V. Meglinski, ${ }^{1, *}$ V.P. Romanov, ${ }^{2}$ D.Y. Churmakov, ${ }^{1}$ E. Berrocal, ${ }^{1}$ M.C. Jermy, ${ }^{1}$ and D.A. Greenhalgh ${ }^{1}$ \\ ${ }^{1}$ School of Engineering, Cranfield University, Cranfield MK43 0AL, UK \\ ${ }^{2}$ Physics Department, St. Petersburg State University, Petrodvoretz 198504, Russia
}

Key words: single-to-multiple scattering; intermediate scattering regime; Monte Carlo method; sprays; scattering orders

PACS: $02.70 . \mathrm{Uu}$, 42.25.Dd, 42.25.Bs, 82.70.Rr, 82.70.-y

The scattering of laser radiation by disperse randomly inhomogeneous scattering media is important in many applications including optical diagnostics in meteorology, powder industries, sprays for combustors, firefighting, drug and agrochemical delivery, and biomedicine. An appropriate single or multiple (diffusing) scattering approximation predicts the propagation of optical radiation in these applications. In single scattering approximation all scattering orders, except the first one describing single scattering, are neglected or/and considered as a perturbation to the single scattering term. In multiple scattering it is assumed that all the terms of series of scattering orders have same order of magnitude, and the diffusion approximation can be applied. Greater complications arise for the intermediate single-to-multiple scattering regime, where the average number of scattering events is too high for single scattering to be assumed, but too low for the diffusion approximation to be applied. This situation is typical, for example, for industrial sprays when making measurements near the nozzle or when the path length of the light in spray medium is long (e.g. in case of high flow mixing systems such as fuel atomizers for large combustors). Accordingly, development of a method operating in the intermediate single-to-multiple regime is strongly required. In this regime both single and higher scattering orders (double, triple, quadruple, etc.) and their contribution to the total intensity should be considered.

In this relation an analysis of different scattering orders in the intermediate scattering regime has been carried out for a randomly inhomogeneous scattering medium with crossed source-detector geometry (Fig. 1). The narrow aperture laser source $S$ illuminates the medium and similar narrow aperture detector $D$ collects diffusely reflected radiation. Detected radiation is localized within the medium in cylindrical volumes $V_{1}$ and $V_{2}$. Due to presence of intermediate integration over the scattering volume analytical expressions for the high scattering orders are complex and involve multi-order integration. However, the Monte Carlo (MC) technique allows us to develop an iterative procedure to determine the contributions of the scattering orders to the total intensity separately, thus, completing the task of experimental data processing.

\footnotetext{
*Corresponding author: e-mail: i.meglinski@ cranfield.ac.uk
} 


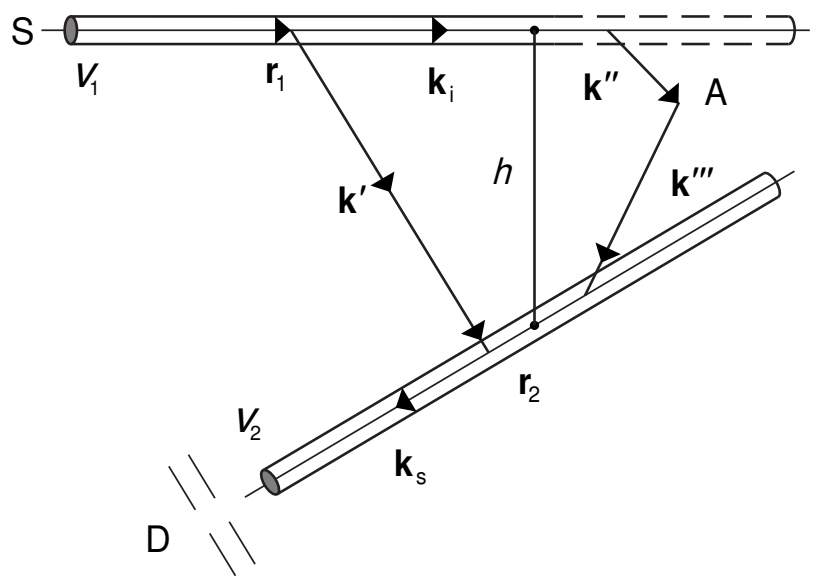

Figure 1 Schematic illustrating the experimental/computational geometry used to account for single-to-higher orders laser scattering. The laser source $S$ and detector $D$ sample two cylindrical volumes, within which single scattering occurs at the points $\mathbf{r}_{1}$ and $\mathbf{r}_{2}$. If the volumes are separated by distance $h$, the detector collects only double and higher orders of scattering. $\mathbf{k}_{i}$, $\mathbf{k}^{\prime}$ and $\mathbf{k}_{s}$ are the wave-vectors of the incident, intermediate and detected double-scattered light, respectively. $\mathbf{k}^{\prime \prime}$ and $\mathbf{k}^{\prime \prime \prime}$ the are intermediate wave vectors for triple light scattering, $A$ is a point of integration over the all scattering volume

The MC algorithm designed for the experimental geometry described in Fig. 1 consists of following steps. Incident radiation is considered normal to the surface of a scattering cube of $50 \mathrm{~mm}$, defined in a 3D coordinate system. The parameters of the cylinders are $L=L_{1}=$ $L_{2}=50 \mathrm{~mm}$. For simplicity the medium is assumed nonabsorbing $\left(\mu_{a}=0\right)$, and homogeneous. Scattering coefficient $\mu_{s}$ is equal to $0.04 \mathrm{~mm}^{-1}$ and corresponds to an average propagation distance of $\langle l\rangle$ equaled to $25 \mathrm{~mm}$ (i.e. single scattering is dominant). The source $S$ is defined by a flat laser beam of $1 \mathrm{~mm}$ diameter, which enters through the middle of the $X Y$ face of the sampling cube. The detector $D$ is characterized by an aperture of $1 \mathrm{~mm}$ diameter located on the top of the cube and positioned at different distances $h$ from the central axis of the sampling cube. The path-length of a photon packet between two scattering events is given by $l_{i}=-\ln \xi_{1} / \mu_{t}$, where $\mu_{t}=\mu_{s}+\mu_{a}$ [1]. After scattering the new direction is specified by the polar and azimuth angles, i.e. by $\theta_{s}$ and $\varphi$, respectively. In this study scattering restricted to isotropic scattering, and $\theta_{s}$ is sampled from $\cos \theta_{s}=2 \xi_{2}-1$, whereas the change of the azimuth angle $\varphi$ is obtained from $\varphi=2 \pi \xi_{3}$. Here $\xi_{1}$, $\xi_{2}$, and $\xi_{3}$ are the random numbers uniformly distributed between 0 and 1 . The probability of photon packet detecting is described as:

$W=p\left(\mathbf{k}_{d}-\mathbf{k}^{\prime}\right) d \Omega_{d} \exp \left(-\mu_{t} d\right)$.

Here, $\mathbf{k}_{d}$ is the vector of normal towards the detector, $d \Omega_{d}$ is the elementary solid angle spanning a line normal to the detector, and $d$ is the distance between the photon scattering and detector. $p\left(\mathbf{k}_{d}-\mathbf{k}^{\prime}\right)$ is the scattering phase function, constant for isotropic scattering: $p\left(\mathbf{k}_{d}-\mathbf{k}^{\prime}\right)=$ $1 / 4 \pi$. The intensity of the scattering orders is obtained then by calculation of (1) for all the scattering events observed by detector (i.e. if direction from scattering center to detector fit the detecting acceptance angle $\theta_{a}$ ). This scheme significantly reduces the computational time (by a factor of $\sim 100$, compare to other MC schemes [2-6]). The intensity of different scattering orders is then determined by the number of photons recorded and can be plotted as a function of $h$.

In order to validate the $\mathrm{MC}$ technique we compare the results of simulation with the results of analytical calculations for double light scattering intensity, described as [7, 8]:

$$
\begin{aligned}
& I_{2}=\frac{I_{0} k_{0}^{8}}{r^{2}(4 \pi)^{4}} \int_{V_{1}} d \mathbf{r}_{1} \int_{V_{2}} d \mathbf{r}_{2} F_{\alpha \beta}\left(\mathbf{k}_{s}, \mathbf{k}_{i}, \mathbf{k}^{\prime}\right) \times \\
& \times \frac{1}{\left|\mathbf{r}_{2}-\mathbf{r}_{1}\right|^{2}} G\left(\mathbf{k}^{\prime}-\mathbf{k}_{i}\right) G\left(\mathbf{k}_{s}-\mathbf{k}^{\prime}\right) \times \\
& \times \exp \left[-\mu_{t}\left(l_{1}+l_{2}+\left|\mathbf{r}_{2}-\mathbf{r}_{1}\right|\right)\right] .
\end{aligned}
$$

Here $I_{0}$ is the intensity of the incident light, $r$ is the distance to the observation point, $k_{0}=\omega / c, \omega$ is the cycle frequency, $c$ is the speed of light in vacuum, $\alpha$ and $\beta$ are the indices of polarization of the incident and scattered light, respectively. $G\left(\mathbf{k}^{\prime}-\mathbf{k}_{i}\right)$ and $G\left(\mathbf{k}_{s}-\mathbf{k}^{\prime}\right)$ are the correlation functions of the permittivity fluctuations [9], $\mathbf{k}^{\prime}-\mathbf{k}_{i}$ and $\mathbf{k}_{s}-\mathbf{k}^{\prime}$ are the wave-vectors of the incident and scattered radiation (see Fig. 1). The exponential multiplier describes attenuation along a path $l_{1}$ before and a path $l_{2}$ after the scattering. $\mu_{t}$ is the extinction coefficient, describing the attenuation of light due to elastic and inelastic interactions of laser radiation within the medium: $\mu_{t}=\mu_{s}+\mu_{a}$.

$$
\begin{aligned}
& F_{\alpha \beta}\left(\mathbf{k}_{s}, \mathbf{k}_{i}, \mathbf{k}^{\prime}\right)=\left(\delta_{\alpha \eta}-k_{\alpha}^{\prime} k_{\eta}^{\prime} / k^{2}\right) \times \\
& \times\left(\delta_{\alpha \nu}-k_{\alpha}^{\prime} k_{\nu}^{\prime} / k^{2}\right)\left(\delta_{\beta \eta}-k_{s \beta} k_{s \eta} / k^{2}\right) \times \\
& \times\left(\delta_{\beta \nu}-k_{s \beta} k_{s \nu} / k^{2}\right)
\end{aligned}
$$

is the polarization factor, where factors

$$
\left(\delta_{\alpha \beta}-k_{\alpha}^{\prime} k_{\beta}^{\prime} / k^{2}\right)
$$

account for the transverse nature of the scattered electromagnetic wave. Summation occurs over the indices (except over $\alpha$ and $\beta$ ) where a quantity has more than one index. Equation (2) describes the intensity of doubly scattered light reaching the detector i.e. singly scattered light entering volume $V_{2}$ and being scattered into the $\mathbf{k}_{s}$ direction with a wave-vector $\mathbf{k}_{s}-\mathbf{k}^{\prime}$. Note, all first order scattering events occur in the volume $V_{1}$ with a wavevector $\mathbf{k}^{\prime}-\mathbf{k}_{i}$, where $\mathbf{k}^{\prime}$ is the intermediate wave-vector (see Fig. 1).

The results of the MC simulation (circles) agree well with the results of calculation by Eq. (2) shown by the 


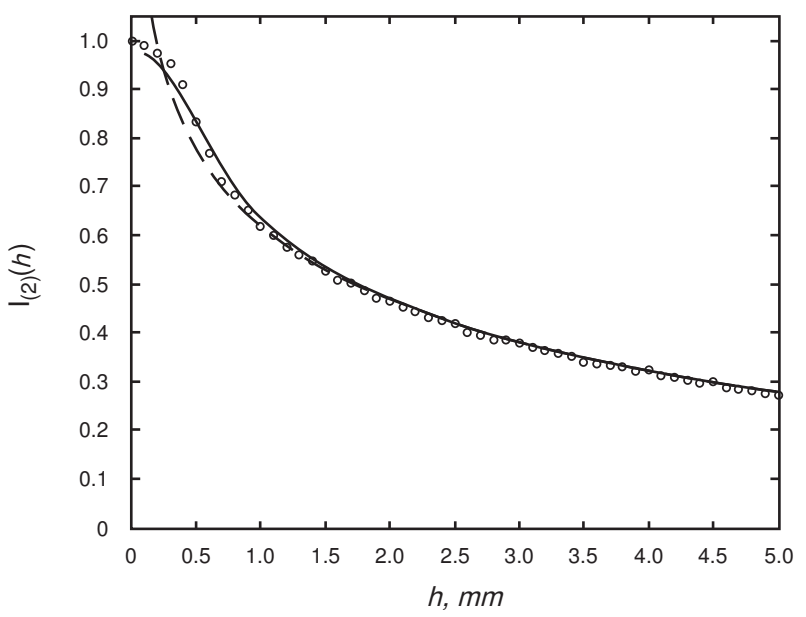

Figure 2 Intensity of double light scattering order with respect to the distance $h$ between $V_{1}$ and $V_{2}$. The solid line represents analytical results obtained by (2), circles show the results of the MC simulation, and dashed line is the results calculated by the long distance approximation

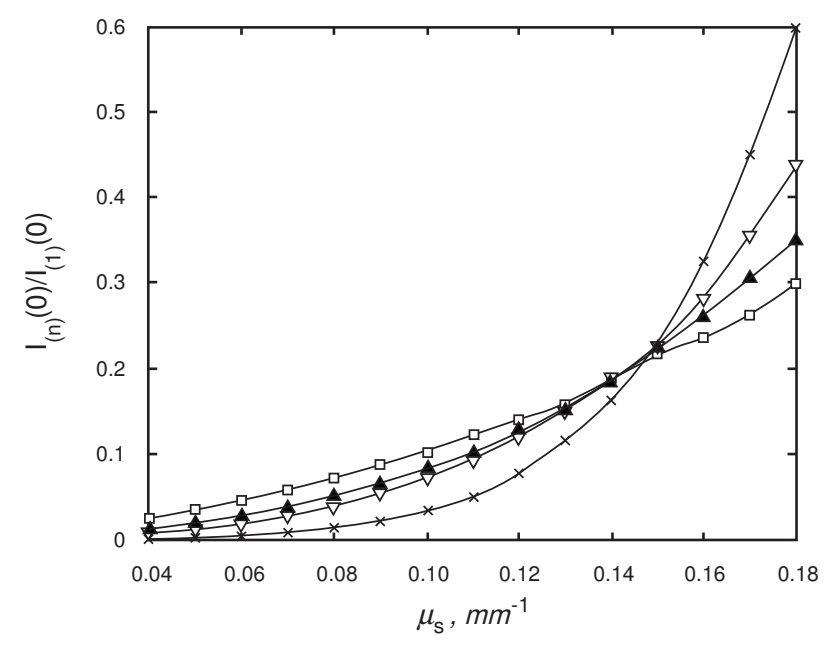

Figure 3 Fraction of high scattering orders versus single scattering as a function of scattering coefficient: $(\square)$ - triple scattering order $I_{(3)},(\boldsymbol{\Lambda})$ - fourth scattering order $I_{(4)},(\nabla)$ - fifth scattering order $I_{(5)}$ and $(\times)$ - tenth scattering order $I_{(10)}$

solid line (Fig. 2). Similar results (dashed line, see Fig. 2) are obtained for a large distances approximation, i.e. when $h \gg R_{1}, R_{2}$. In that case Eq. (2) is significantly simplified and can be written as:

$$
\begin{aligned}
& I_{2}(h)=A \pi^{2} R_{1}^{2} R_{2}^{2} \int_{-L_{1} / 2}^{L_{1} / 2} d x \int_{-L_{2} / 2}^{L_{2} / 2} d z \frac{1}{x^{2}+z^{2}+h^{2}} \times \\
& \times \exp \left[-\mu_{t}\left(L+z-x+\sqrt{x^{2}+z^{2}+h^{2}}\right)\right] .
\end{aligned}
$$

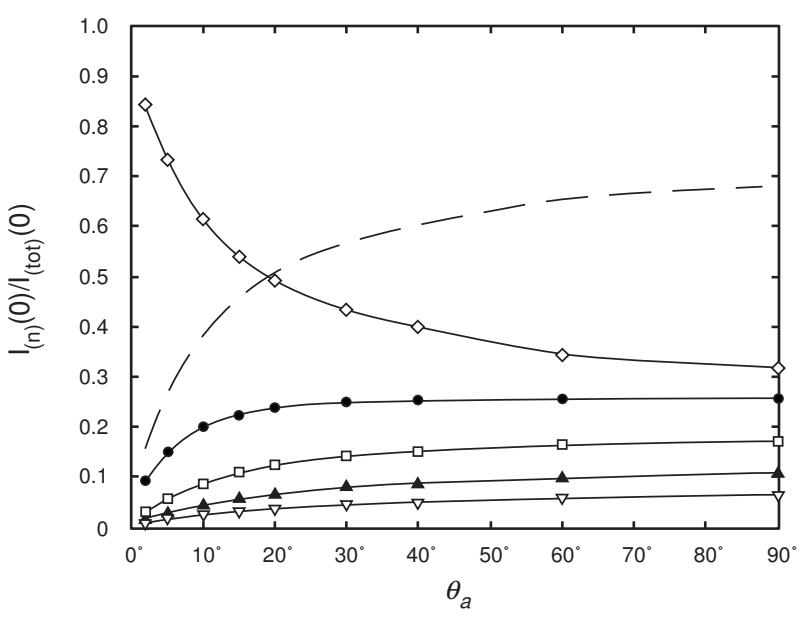

Figure 4 Results of MC calculation of the different scattering order intensities $I_{(n)} / I_{(t o t)}$ versus detector angular aperture: $(\diamond)$ - single scattering $I_{(1)},(\bullet)$ - double scattering $I_{(2)},(\square)$ - triple scattering $I_{(3)},(\boldsymbol{\Lambda})$ - fourth order scattering $I_{(4)},(\nabla)$ fifth order scattering $I_{(5)}$. The dashed line represents the relative contribution of multiple scattering to the total intensity, $\left(I_{(t o t)}-I_{(1)}\right) / I_{(t o t)}$

Here, $L+z-x=l_{1}+l_{2} ; R_{1}$ and $R_{2}$ are the radii of $V_{1}$ and $V_{2}$ cylinders directed along the axes $Z$ and $X$, respectively. The mismatching between the exact (2) and approximate (4) formulas begins when $h$ is close to the diameter of cylinders (see Fig. 2).

Fig. 3 illustrates a ratio of high orders of scattering to single scattering intensity at the $h=0$, i.e. $I_{(n)}(0) / I_{1}(0)$, where $n=\{3-5,10\}$. This ratio monotonically increases with the increasing scattering order. When $\mu_{s}=$ $0.14-0.15 \mathrm{~mm}^{-1}$ the values of $I_{(n)} / I_{1}$ are close, and for higher $\mu_{s}$ an inversion of scattering orders takes place (see Fig. 3). This interval illustrates the transition from single to multiple scattering. It is clear that contribution of high scattering orders is significant, about $15-20 \%$ of the single scattering intensity at the $h=0$ (see Fig. 3) when $\mu_{s} \sim 0.15 \mathrm{~mm}^{-1}$. The obtained results agreed well with recently reported experimental data [10].

Figs. 4 and 5 present the results of a study of the detection parameters. The results of simulation of scattering orders calculated by the $\mathrm{MC}$ technique for the scattering medium with $\mu_{s}=0.04 \mathrm{~mm}^{-1}$ are represented in Fig. 4 as a function of numerical aperture. Single scattering, that occurs only in $V_{1}$ and $V_{2}$ intersection (see Fig. 1), dominates at small $\theta_{a}\left(\theta_{a}<20^{\circ}\right)$, where the contribution of the other scattering orders is relatively low. The dashed line represents the total intensity of high orders calculated separately from the single scattering.

The intensity of the different scattering orders versus numerical aperture is shown in Fig.5. It is seen that the dependence is more pronounced for single and double light scatterings (see Fig. $5 \mathrm{a}$ and b). The high scattering orders 

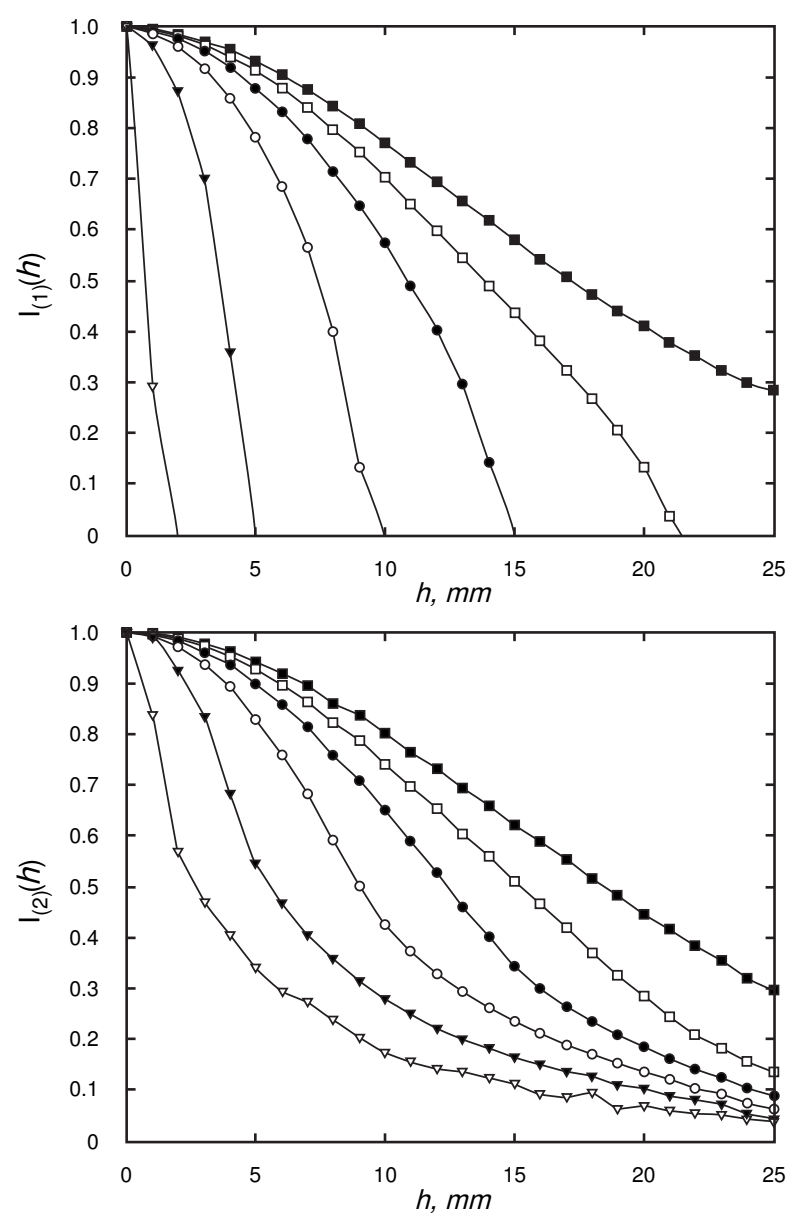

Figure 5 Normalized intensity of different scattering orders versus $h$ : a) single light scattering; b) double scattering; c) triple scattering; d) fifth order scattering. The symbols represents different values of the detector numerical aperture: $(\nabla)-2^{\circ},(\boldsymbol{\nabla})$ $10^{\circ},(\circ)-20^{\circ},(\bullet)-40^{\circ},(\square)-60^{\circ},(\square)-90^{\circ}$

(e.g. third and fifth) are independent of the detecting angle $\theta_{a}$ (see Fig. $5 \mathrm{c}$ and d). This is explained by the fact that, the localization of mean path-length of the photon packets between source and detector is more critical for single and double scattering, and less for the high orders of scattering.

The developed MC simulations allow us to develop an iterative procedure to determine the contributions of the higher scattering orders (triple, quadruple etc. scattering) to the total intensity separately, thus, completing the task of experimental data processing. Both analytical and MC calculations show a strong logarithmic dependence of double light scattering from the distance $h$ between illuminating and collecting volumes. Dependence of higher scattering orders is weaker and can be considered constant compared to the single order. The considered geometry (Fig. 1) allows one to separate single scattering from high orders of scattering. It is then possible to extract information on the absolute values of the medium optical properties from the ratio of single to double scattering intensity. If the double and single light scattering intensity are of the same order of magnitude intensity then the higher order scattering, $I_{(n)}, n \geq 3$, should be taken into account.

In addition, the results support the use of the MC approach in the intermediate scattering regime $(0.04 \leq$ $\left.\mu_{s} \leq 0.16 \mathrm{~mm}^{-1}\right)$, and provide details of transition from low scattering to multiple scattering. The method used can be applied to verify the analytical results indirectly against experiment via MC calculations that include the imperfections of the experiment. The procedure developed can readily be generalized for a case of anisotropic scatters, spatially inhomogeneous media and dispersed systems composed of particles of a different size. We intend to apply the MC code verified here to the estimation and suppression of errors in existing spray diagnostics and the development of new spray diagnostics.

Acknowledgements The authors (IVM, VPR and MCJ) acknowledge support from The Royal Society (Project 15298) and NATO (Project PST.CLG.979652), EB, MCJ and DAG also acknowledge support of EPSRC (grant GR/R92653), and DYC would like to thank the British Overseas Research Scholarships scheme for support.

\section{References}

[1] I.M. Sobol', The Monte Carlo Method (The University of Chicago Press, Chicago, 1974).

[2] V.P. Kandidov, Usp. Fiz. Nauk 39, 1243 (1996).

[3] I.V. Meglinskii and S.J. Matcher, Opt. Spectr. 91, 654 (2001).

[4] D.Y. Churmakov, I.V. Meglinski, and D.A. Greenhalgh, Phys. Med. Biol. 47, 4271 (2002).

[5] V.L. Kuzmin and I.V. Meglinski, JETP Letters 79, 109 (2004).

[6] S.V. Gangnus, S.J. Matcher, and I.V. Meglinski, Laser Phys. 14, (2004).

[7] V.L. Kuz'min and V.P. Romanov, Usp. Fiz. Nauk 39, 231 (1996).

[8] V.L. Kuzmin, V.P. Romanov, and L.A. Zubkov, Phys. Reports 248, 71 (1994).

[9] V.L. Kuzmin, V.P. Romanov, and I.V. Meglinski, Opt. Spectr. 96, 106 (2004).

[10] K.K. Bizheva, A.M. Siegel, and D.A. Boas, Phys. Rev. E 58, 7664 (1998). 